\title{
Uma Implementação de Pontos Interiores para Fluxo em Redes Lineares por Partes
}

C. PERIN 1 , M. MELLO ${ }^{2}$, Departamento de Matemática Aplicada, IMECC, Universidade Estadual de Campinas-UNICAMP, UNICAMP, Cx.P. 6065, 13083200 Campinas, SP, Brasil

F.A.S. MARINS ${ }^{3}$, Departamento de Engenharia de Produção, Faculdade de Engenharia de Guaratinguetá, Universidade Estadual Paulista-UNESP, Cx.P. 205, 12500-000 Guaratinguetá, SP, Brasil.

\begin{abstract}
Resumo. Problemas de Fluxo em Redes Lineares por Partes constituem uma importante área de aplicação, onde são estudados modelos que tratam da minimização de uma função objetivo convexa, separável e linear por partes, sujeita a um sistema de equações lineares que expressam a conservação de fluxo sobre uma rede conectada. Este problema pode ser transformado em um programa linear equivalente de tamanho maior. Neste trabalho apresentamos e comparamos duas implementações do método primal-dual para redes: uma para problemas lineares e outra para problemas lineares por partes. Ambas as implementações utilizam estruturas de dados especiais visando diminuir o gasto com memória e tempo. A estrutura utilizada para redes lineares por partes pode ser considerada uma extensão daquela utilizada em redes lineares. Cabe observar que o esforço computacional de cada iteração está concentrado na construção e resolução de um sistema linear quadrado, simétrico e definido positivo, resolvido por um método de gradientes conjugados pré-condicionado. Além disto, a matriz do sistema não é construída explicitamente. Foram realizados diversos testes computacionais que permitem concluir afirmativamente a respeito da vantagem computacional da segunda implementação.
\end{abstract}

\section{Introdução}

Considera-se um problema de minimização com função objetivo convexa, separável e linear por partes, e com restrições que representam a conservação de fluxo em uma rede conectada. Este modelo pode surgir em um contexto teórico, quando se estudam métodos para obtenção de solução inicial para problemas lineares, ou diretamente de aplicações práticas, por exemplo nas áreas de telecomunicações,

\footnotetext{
${ }^{1}$ clovis@ime.unicamp.br

2 margarid@ime.unicamp.br

3 fmarins@feg.unesp.br
} 
planejamento de operação de usinas hidrelétricas e distribuição de energia elétrica. No primeiro caso a rede matemática é um retrato direto da rede telefônica e a quantidade de fluxo que trafega por um arco corresponde à intensidade de tráfego telefônico entre os nós ligados por este arco. Como neste tipo de problema geralmente admite-se a possibilidade de expansão de capacidade de determinados arcos, é natural modelar esta situação atribuindo-se uma estrutura de custos linear por partes para os arcos. Assim, os custos são lineares para determinadas faixas de fluxo com coeficientes maiores para as faixas mais elevadas. A importância e a ampla utilização destes modelos decorrem das propriedades de facilidade de visualização da solução, flexibilidade dos modelos e disponibilidade de métodos eficientes para resolvê-los. Para tanto têm sido desenvolvidas estruturas de dados específicas e algoritmos de programação linear têm sido adaptados. Em particular, o método simplex para redes, que já é uma adaptação eficiente do método simplex para programas lineares, foi adaptado com sucesso para redes lineares por partes, ver $[3,6]$. Após o advento dos algoritmos de pontos interiores para programação linear, e.g. [9], surgiram iniciativas no sentido de formular uma variante eficiente para problemas em redes, cf. [11]. Neste trabalho desenvolvemos uma adaptação bem sucedida de pontos interiores para redes lineares por partes, considerando uma estrutura especial de dados, prosseguindo com trabalho já realizado em $[7,8,10]$.

O custo linear por partes de uma variável é expresso por meio de duas seqüências crescentes de números: a primeira contendo os extremos dos intervalos consecutivos dentro dos quais o custo é linear e a segunda com os coeficientes de custo para cada intervalo. Ho, em [4], apresenta os quatro métodos mais usuais para transformar um problema linear por partes em um problema linear equivalente, trabalhando cada variável cujo custo é linear por partes da seguinte forma:

- no $\delta$-Método (sum-of-intervals ou bounded variables representation) a variável é substituída pela soma de outras variáveis, uma para cada intervalo e o custo associado à variável original é substituído por uma combinação linear das novas variáveis cujos multiplicadores são os coeficientes de custo dos intervalos correspondentes;

- no $\lambda$-Método (weighted-grid-point ou inner approximation) a variável é substituída pela combinação convexa dos extremos dos intervalos e o custo associado à variável original é substituído pela mesma combinação convexa dos custos correspontes aos extremos dos intervalos;

- o $\sigma$-Método (outer approximation) utiliza o fato que uma função convexa e linear por partes pode ser expressa como o máximo pontual de funções lineares

- no $\gamma$-Método (sum-of-projections representation) o custo é calculado com o efeito cascata, conhecido por todos os declarantes de IR.

Os autores [10] compararam o uso dessas transformações em problemas em redes lineares por partes. O método mais promissor, segundo este estudo, foi o $\delta$-Método. Ademais, este é o único método que preserva a estrutura de rede da matriz de coeficientes. 
Conseqüentemente, este foi o método adotado para comparar as duas abordagens de resolução via pontos interiores: (i) transformando-se o problema linear por partes em um problema linear equivalente e aplicando-se a este último um método de pontos interiores para redes lineares e (ii) resolvendo-se o problema linear por partes por uma variante de método de pontos interiores capaz de lidar diretamente, sem a necessidade de transformações, com redes lineares por partes.

Este trabalho insere-se, portanto, em uma linha de pesquisa mais ampla, na qual são exploradas várias facetas das possíveis abordagens do problema de redes lineares por partes: os tipos de algoritmos que podem ser utilizados, as transformações, as estruturas de dados, os detalhes de implementação relevantes (e.g., rotinas para a resolução de sistemas lineares), etc., com o objetivo de se determinar qual a abordagem ideal para este tipo de problema.

O trabalho apresenta-se organizado como segue: na seção 2. o arcabouço dos algoritmos de pontos interiores implementados é descrito, na seção 3. discutem-se os detalhes das implementações descrevendo-se as estruturas de dados utilizadas. Os testes computacionais são relatados na seção 4. e as conclusões e direções para trabalhos futuros são resumidas na seção 5 .

\section{Desenvolvimento e Descrição do Algoritmo}

Neste trabalho mostramos que é interessante considerar a implementação especializada de um método de pontos interiores para problemas de fluxo em redes lineareas por partes ao invés de considerar a sua transformação em um problema de fluxo em redes lineares.

Diversos métodos de primais-duais de pontos interiores já foram propostos e os pontos centrais de discussão podem ser resumidos em: (1) A cada iteração primaldual, resolver um sistema simétrico e definido positivo de equações lineares ou resolver o sistema aumentado equivalente? (2) No caso de resolver o sistema simétrico e definido positivo, utilizar a fatoração de Cholesky, Gradientes Conjugados, ou algum outro método? (3) No caso de precondicionamento para o método dos gradientes conjugados utilizar o precondicionador diagonal, da árvore geradora máxima, ou algum outro?

Diversos autores, e.g. [11], mostraram que é eficiente resolver o problema de fluxo em redes lineares computando, a cada iteração do método primal-dual de pontos interiores, a solução de um sistema simétrico e definido positivo com o método dos gradientes conjugados precondicionado pelo precondicionador diagonal, nas iterações iniciais, e pelo precondicionador da árvore geradora máxima, nas iterações subseqüentes do método primal-dual. Vamos utilizar esta mesma metodologia para resolver problemas de fluxo em redes lineares por partes.

O problema de fluxo em redes lineares por partes definido por

$$
\min \{f(x) \mid A x=b, x \geq 0\}
$$

onde $A$ é a $n \times m$-matriz de incidência de uma rede com $n$ nós e $m$ arcos, $b$ é o $n$ vetor de demandas dos nós, $x$ é um $m$-vetor de fluxos nos arcos, a ser determinado, e 
$f(x)=\sum_{j=1}^{m} f_{j}\left(x_{j}\right)$ é uma função convexa, separável e linear por partes. As funções convexas e lineares por partes $f_{j}$ podem ser especificadas pelo par de $\ell$-vetores $c, d$, onde $\ell=\sum_{j=1}^{m} \ell_{j}$ é o número total de intervalos, sendo $\ell_{j}$ o número de intervalos de $f_{j}$.

Este problema pode ser transformado em um problema de fluxo em redes lineares definido por

$$
\min \left\{c^{\prime} \bar{x} \mid \bar{A} \bar{x}=b, \bar{x}+\bar{s}=d, \bar{x}, \bar{s} \geq 0\right\}
$$

Neste caso, a $n \times \ell$-matriz $\bar{A}$ é obtida a partir da matriz $A$ com a replicação de cada uma de suas colunas tantas vezes quantos são os intervalos a ela associados. Estamos supondo que esta replicação também é feita no vetor $x$ transformando-o no vetor $\bar{x}$. O problema dual associado é dado por

$$
\max \left\{b^{\prime} y-d^{\prime} \bar{w} \mid \bar{A}^{\prime} y-\bar{z}+\bar{w}=c, \bar{z}, \bar{w} \geq 0\right\}
$$

e as condições de folgas complementares são expressas por

$$
\bar{X} \bar{Z} 1=0 \quad \bar{S} \bar{W} \mathbf{1}=0
$$

onde $\bar{X}, \bar{S}, \bar{Z}, \bar{W}$ são matrizes diagonais com os elementos de $\bar{x}, \bar{s}, \bar{z}, \bar{w}$, respectivamente, e 1 é um vetor de 1's.

O método primal-dual de pontos interiores adotado é inicializado a partir de um iterando $\bar{x}, \bar{s}, y, \bar{z}, \bar{w}$ satisfazendo $\bar{x}, \bar{s}, \bar{z}, \bar{w}>0$ e a cada iteração é obtida uma direção de deslocamento $\Delta \bar{x}, \Delta \bar{s}, \Delta y, \Delta \bar{z}, \Delta \bar{w}$ em conformidade com um parâmetro de centragem $\mu$ de acordo com as equações abaixo que refletem a factibilidade primal, a factibilidade dual, e a complementaridade das soluções quando $\mu=0$.

$$
\begin{aligned}
\bar{A} \Delta x & =b-\bar{A} \bar{x} \\
\Delta \bar{x}+\Delta \bar{s} & =d-\bar{x}-\bar{s} \\
\bar{A}^{\prime} \Delta y+\Delta \bar{z}-\Delta \bar{w} & =c-\bar{A}^{\prime} y-\bar{z}+\bar{w} \\
\bar{X} \Delta \bar{z}+\bar{Z} \Delta \bar{x} & =\mu 1-\bar{X} \bar{z} \\
\bar{S} \Delta \bar{w}+\bar{W} \Delta \bar{s} & =\mu 1-\bar{S} \bar{w}
\end{aligned}
$$

Isto resulta na seguinte seqüência de comandos:

$$
\begin{aligned}
D & \leftarrow\left(\bar{X}^{-1} \bar{Z}+\bar{S}^{-1} \bar{W}\right)^{-1} \\
Q & \leftarrow \bar{A} D \bar{A}^{\prime} \\
q & \leftarrow b-\bar{A} \bar{x}+\bar{A} D\left(c-\bar{A}^{\prime} y-\mu\left(\bar{X}^{-1}-\bar{S}^{-1}\right) 1-\bar{S}^{-1} \bar{W}(d-\bar{x}-\bar{s})\right) \\
\Delta y & \leftarrow \text { solução do sistema } Q \Delta y=q \\
\Delta \bar{x} & \leftarrow D\left(\bar{A}^{\prime} \Delta y-c+\bar{A}^{\prime} y+\mu\left(\bar{X}^{-1}-\bar{S}^{-1}\right) 1+\bar{S}^{-1} \bar{W}(d-\bar{x}-\bar{s})\right) \\
\Delta \bar{s} & \leftarrow d-\bar{x}-\bar{s}-\Delta \bar{x} \\
\Delta \bar{z} & \leftarrow \mu \bar{X}^{-1} 1-\bar{z}-\bar{X}^{-1} \bar{Z} \Delta \bar{x} \\
\Delta \bar{w} & \leftarrow \mu \bar{S}^{-1} 1-\bar{w}-\bar{S}^{-1} \bar{W} \Delta \bar{s}
\end{aligned}
$$


O algoritmo pára quando uma das seguintes condições é satisfeita: (1) número máximo de iterações é excedido; (2) soluções quase-complementares $\bar{x}^{\prime} \bar{z}+\bar{s}^{\prime} \bar{w} \approx 0$ são encontradas; (3) limite inferior para o valor da função objetivo primal é excedido; (4) limite superior para o valor da função objetivo dual é excedido. O parâmetro $\mu$ é atualizado dividindo-se a complementaridade média corrente pelo número de variáveis.

O método primal-dual de pontos interiores pode ser esquematizado da seguinte forma:

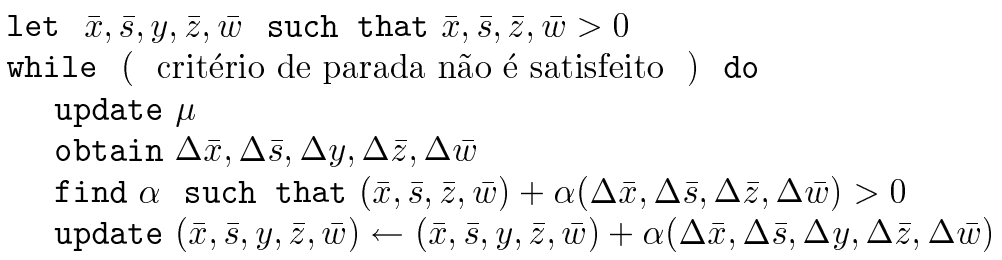

Destacamos a seguir os aspectos que são diferentes entre as duas versões que são implementadas do algoritmo, para redes lineares e para redes lineares por partes. $\mathrm{Na}$ especialização do algoritmo para redes lineares por partes trabalha-se com um vetor adicional, um vetor de escala para os arcos, além do vetor de escala para os intervalos, com vistas a reduzir o esforço computacional. Na comparação abaixo veremos como o uso deste vetor permite reduzir o número de operações, enfocando, em particular, o esforço dispendido na execução do gradiente conjugado, que é efetuada a cada iteração:

(1) O produto $u \leftarrow Q v$ é computado em cada iteração do método do gradiente conjugado precondicionado para a resolução do sistema $Q \Delta y=q$. A matriz simétrica e definida positiva $Q=\bar{A} D \bar{A}^{\prime}$ é manipulada implicitamente da seguinte forma: o vetor $u$, que é inicializado com zeros, é construído somandose parcelas apropriadas, uma por arco (para redes lineares por partes) ou por intervalo (para redes lineares). No caso de redes lineares por partes, obtém-se a diferença entre as componentes de $v$ da cabeça e da cauda de cada arco, multiplica-se esta diferença pelo fator de escala do arco e soma-se na componente de $u$ da cabeça do arco e subtrai-se na componente de $u$ da cauda do arco. No caso de redes lineares, faz-se a mesma operação, porém todas as réplicas dos arcos devem ser consideradas, portanto ao invés de lidarmos com $m$ arcos, estaremos lidando com $\ell$ réplicas de arcos.

(2) O precondicionador da árvore geradora máxima nas aplicações do método do gradiente conjugado com este precondicionador é determindado da seguinte forma: constrói-se o monte (head) de ordenação dos arcos (ou intervalos) de acordo com o seu fator de escala e retira-se do monte os arcos (ou intervalos) de maior fator de escala até se obter uma árvore geradora. Esta árvore corresponde à base de uma solução extrema próxima da solução corrente e tem a propriedade de ser triangularizável. Note-se que a dimensão do monte para redes lineares é de $\ell$ elementos (intervalos ou réplicas de arcos) enquanto que, para redes lineares por partes, o monte tem dimensão $m$. 
Cabe observar que nas duas implementações, para redes lineares e para redes lineares por partes, trabalhamos com um vetor solução $\ell$ dimensional $(\bar{x})$. Outros trabalhos, e.g. [1], adotam estratégia diferente, trabalhando com vetor solução $m$ dimensional.

\section{Implementação}

Os programas foram implementados em $\mathrm{C}$ e os testes computacionais foram realizados em uma estação de trabalho SPARCStation 5. A estrutura de dados utilizada na implementação para pontos interiores utiliza vetores de tamanho definidos por $n$ nós, $m$ arcos e $\ell$ intervalos:

- armazenamento da rede linear: $1 n$-vetor para armazenar $b, 2 \ell$-vetores, para armazenar a cabeça e a cauda de cada réplica de arco e 2 -vetores para armazenar $c, d$.

- armazenamento da rede linear por partes: $1 n$-vetor para armazenar $b, 2 m$ vetores para armazenar a cabeça e a cauda de cada arco e 3 l-vetores para armazenar $c, d$ e o arco de cada intervalo.

- armazenamento da solução e variáveis auxiliares: $2 n$-vetores para armazenar os vetores $y, \Delta y, \hat{b}, 10 \ell$-vetores para armazenar $\bar{x}, \bar{s}, \bar{z}, \bar{w}, \Delta \bar{x}, \Delta \bar{s}, \Delta \bar{z}, \Delta \bar{w}, \hat{c}$, $d^{\ell}$ e, no caso de redes lineares por partes, mais $1 m$-vetor para armazenar $d^{m}$, o fator de escala de cada arco. Os vetores $\bar{s}, \bar{w}, \Delta \bar{s}, \Delta \bar{w}$ têm a sua dimensão reduzida para o número de intervalos com canalização finita.

- variáveis auxiliares para o gradiente conjugado: 4 n-vetores para armazenar a solução, o resíduo, a dire cão de deslocamento da solução e a direção de redução do resíduo.

- precondicionamento diagonal: $1 n$-vetor para armazenar $d^{n}$.

- precondicionamento da árvore geradora máxima: 4 -vetores para armazenar o predecessor, fio (thread), fio reverso e sentinela de nó, e $1 \mathrm{~m}$-vetor para armazenar o monte (heap) de ordenação.

- Obs.: $\hat{b}=b-A x, \hat{c}=c-A^{\prime} y-z+w$, $d^{\ell}$ é o fator de escala de cada intervalo, $d^{m}$ é o fator de escala de cada arco otido com a soma dos fatores de escala de seus intervalos e $d^{n}$ é o fator de escala de cada nó obtido com a soma dos fatores de escala dos arcos incidentes.

As tolerâncias utilizadas, em ambas as implementações, foram: $10^{-8}$ (para tolerância relativa no teste de complementaridade), $-10^{8}$ (para limite inferior da função objetivo primal) e $10^{8}$ (para limite superior da função objetivo dual). O número máximo de iterações, em ambas as implementações, é de 100. 


\section{Testes Computacionais}

Foram geradas aleatoriamente redes conectadas com 1000 a 10000 nós, 2000 a 50000 arcos e 4000 a 224000 intervalos. Os tempos de CPU (em segundos) e o número de iterações gasto por cada programa para cada rede estão relacionados nas Tabelas 1 , 2, 3, 4 e 5, onde a terminação "L" está associada ao algoritmo para redes lineares e a terminação "P" ao algoritmo para redes lineares por partes. Estes números correspondem a médias, foram gerados 3 exemplares factíveis para cada tamanho de rede (nós, arcos e intervalos). É possível observar que, embora o número de iterações não apresente grandes diferenças, o tempo de CPU é sensivelmente menor para o algoritmo adaptado para redes lineares por partes. A média das taxas cpuL/cpuP para todos os testes é aproximadamente 1,5, ou seja, a estratégia de resolução que consiste em resolver o problema linear equivalente resulta em aproximadamente $50 \%$ a mais de tempo computacional para a resolução do problema.

\begin{tabular}{rr|rr|rr}
\hline no. arcos & no. interv. & cpuL & cpuP & itrL & itrP \\
\hline 2000 & 4000 & 65 & 51 & 65 & 65 \\
2000 & 10000 & 65 & 43 & 49 & 49 \\
2000 & 16000 & 72 & 42 & 42 & 42 \\
3500 & 7000 & 64 & 48 & 53 & 53 \\
3500 & 17500 & 75 & 46 & 40 & 40 \\
3500 & 28000 & 98 & 53 & 38 & 38 \\
5000 & 10000 & 65 & 47 & 45 & 45 \\
5000 & 25000 & 93 & 54 & 39 & 39 \\
5000 & 40000 & 130 & 69 & 39 & 39 \\
\hline
\end{tabular}

Tabela 1: Resultados experimentais para redes com 1000 nós

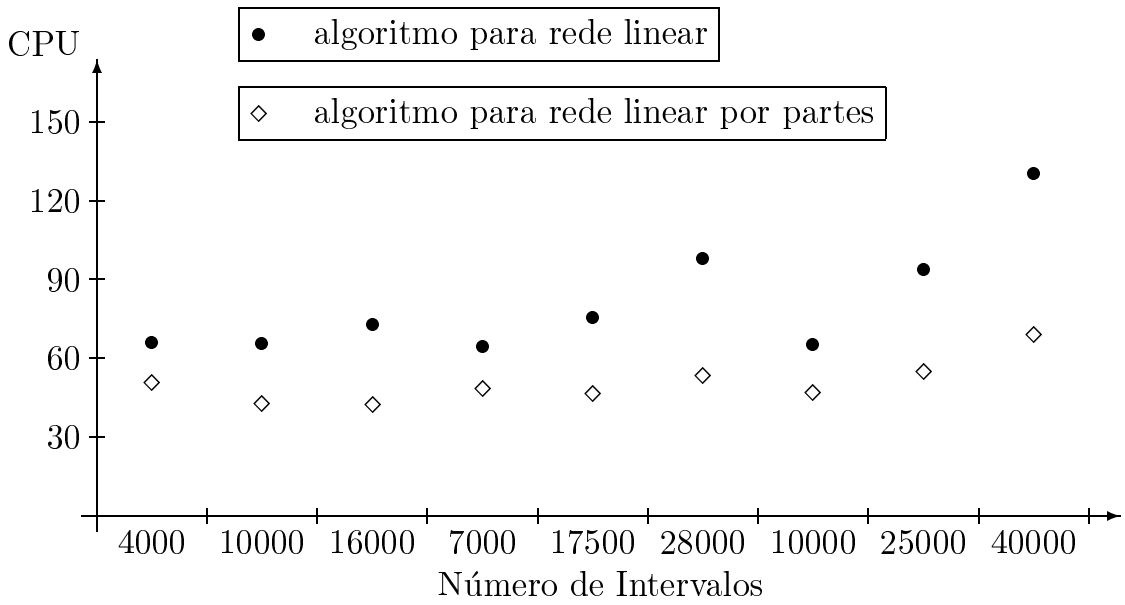

Figura 1: Tempos de cpu para redes com 1000 nós 
Os dados da Tabela 1 referentes aos tempos de CPU estão organizados em forma de gráfico na figura 1. Esta representação gráfica pretende facilitar a percepção da diferença relativa entre os tempos.

\begin{tabular}{rr|rr|rr}
\hline no. arcos & no. interv. & cpuL & cpuP & itrL & itrP \\
\hline 4000 & 8000 & 240 & 195 & 79 & 79 \\
4000 & 20000 & 215 & 148 & 56 & 56 \\
4000 & 32000 & 219 & 137 & 48 & 48 \\
7000 & 14000 & 224 & 175 & 63 & 63 \\
7000 & 35000 & 216 & 138 & 44 & 44 \\
7000 & 56000 & 275 & 157 & 43 & 43 \\
10000 & 20000 & 226 & 167 & 54 & 54 \\
10000 & 50000 & 260 & 158 & 42 & 42 \\
10000 & 80000 & 373 & 196 & 43 & 43 \\
\hline
\end{tabular}

Tabela 2: Resultados experimentais para redes com 2000 nós

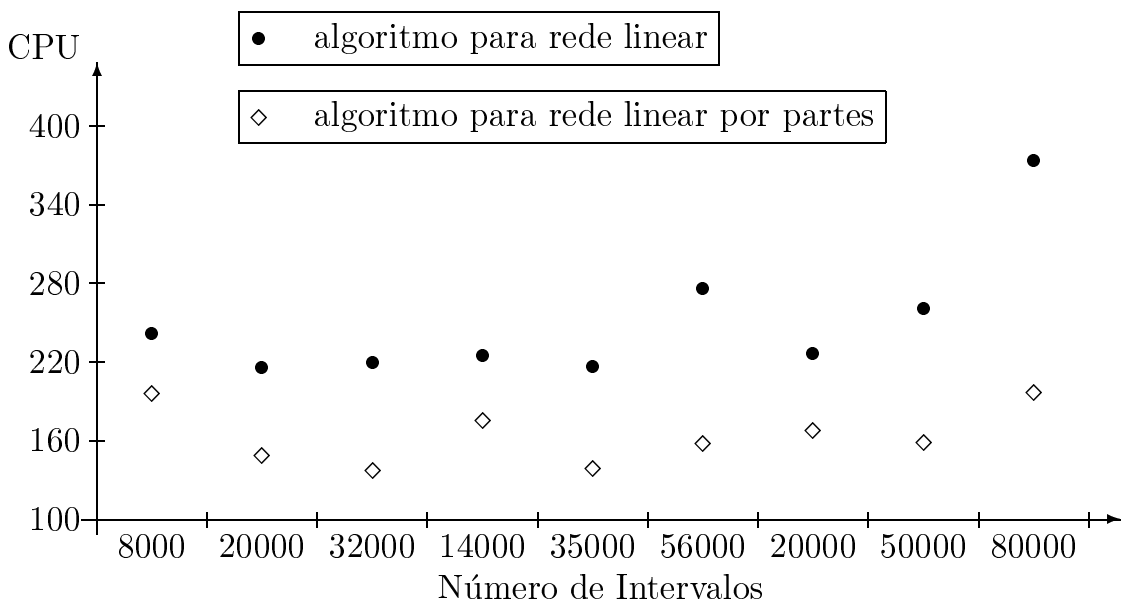

Figura 2: Tempos de cpu para redes com 2000 nós

Convém observar que, como era de se esperar, a superioridade da estratégia de resolver o problema por um algoritmo especializado para redes lineares por partes, fica mais evidente quanto maior o número de intervalos. Se comparamos os tempos de CPU para redes com mesmo número de arcos, porém com número crescente de intervalos (note que há três números de intervalos para cada número de arcos), vemos que a distância entre o círculo cheio "•" e o losango " $\diamond$ " fica maior. Este fenômeno está presente em todos os gráficos. 


\begin{tabular}{rr|rr|rr}
\hline no. arcos & no. interv. & cpuL & cpuP & itrL & itrP \\
\hline 8000 & 16000 & 948 & 788 & 92 & 92 \\
8000 & 40000 & 774 & 570 & 67 & 67 \\
8000 & 64000 & 748 & 494 & 55 & 55 \\
14000 & 28000 & 862 & 697 & 76 & 76 \\
14000 & 70000 & 699 & 478 & 50 & 50 \\
14000 & 112000 & 780 & 468 & 44 & 44 \\
20000 & 40000 & 795 & 618 & 63 & 63 \\
20000 & 100000 & 821 & 504 & 46 & 46 \\
20000 & 160000 & 1068 & 592 & 48 & 48 \\
\hline
\end{tabular}

Tabela 3: Resultados experimentais para redes com 4000 nós

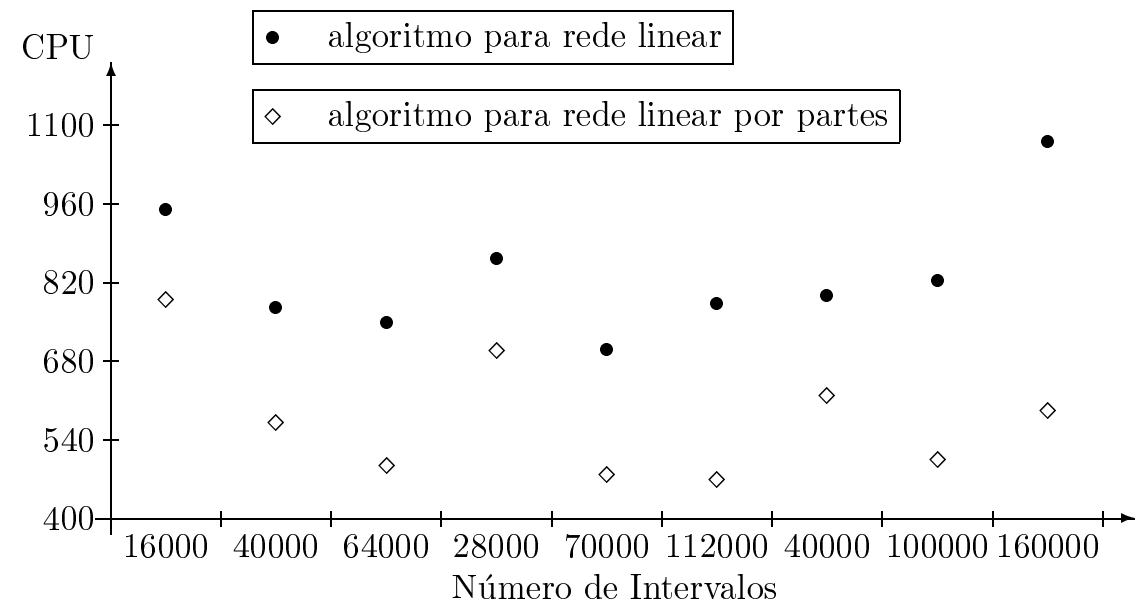

Figura 3: Tempos de cpu para redes com 4000 nós

\begin{tabular}{rr|rr|rr}
\hline no. $\operatorname{arcos}$ & no. interv. & cpuL & cpuP & itrL & itrP \\
\hline 16000 & 128000 & 2875 & 2051 & 67 & 67 \\
28000 & 56000 & 3338 & 2837 & 90 & 90 \\
28000 & 140000 & 2585 & 1856 & 57 & 57 \\
28000 & 224000 & 2606 & 1679 & 50 & 50 \\
40000 & 80000 & 2909 & 2351 & 71 & 71 \\
40000 & 200000 & 2699 & 1807 & 51 & 51 \\
\hline
\end{tabular}

Tabela 4: Resultados experimentais para redes com 8000 nós 


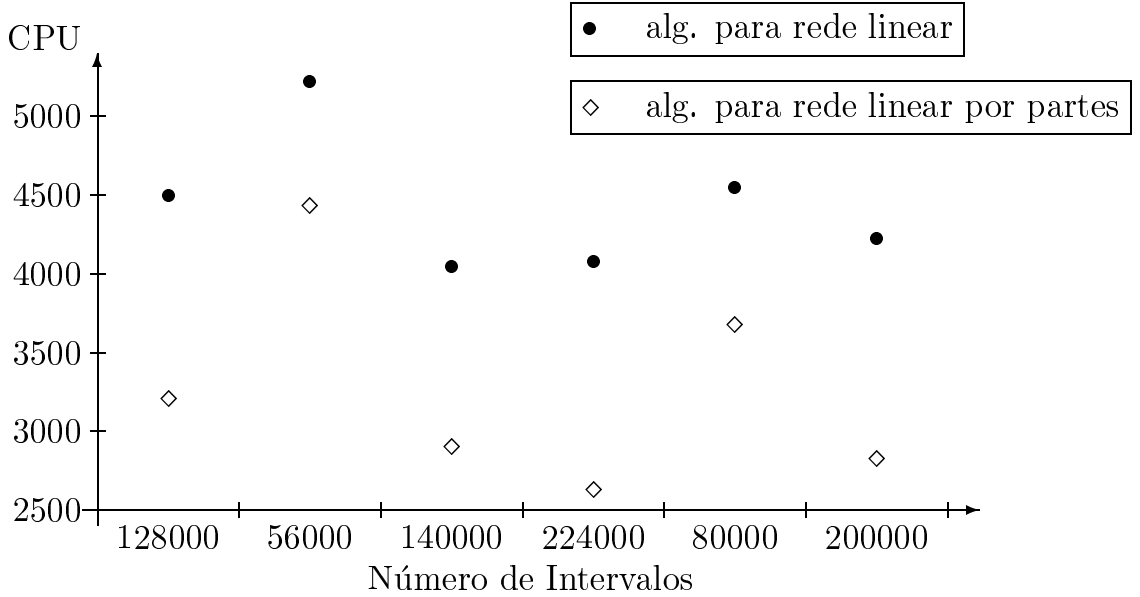

Figura 4: Tempos de cpu para redes com 8000 nós

\begin{tabular}{rr|rr|rr}
\hline no. arcos & no. interv. & cpuL & cpuP & itrL & itrP \\
\hline 20000 & 100000 & 4647 & 3602 & 80 & 80 \\
20000 & 160000 & 4334 & 3201 & 69 & 69 \\
35000 & 70000 & 4940 & 4236 & 88 & 88 \\
35000 & 175000 & 3920 & 2933 & 61 & 61 \\
50000 & 100000 & 4615 & 3768 & 77 & 77 \\
\hline
\end{tabular}

Tabela 5: Resultados experimentais para redes com 10000 nós

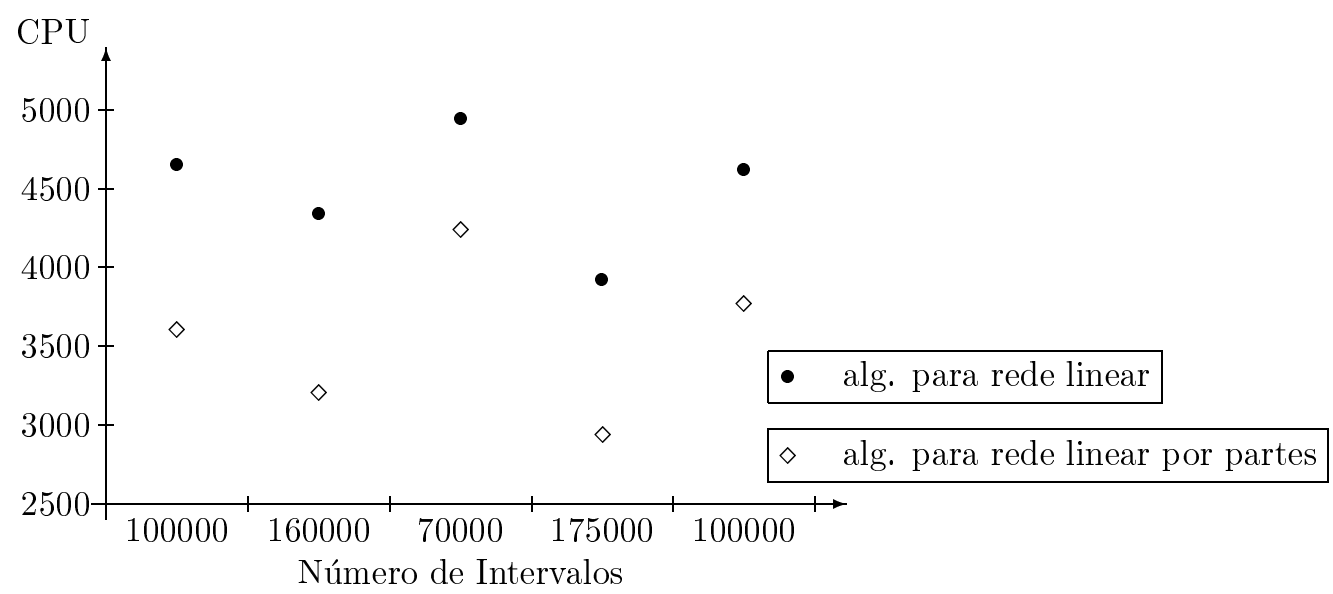

Figura 5: Tempos de cpu para redes com 10000 nós 


\section{Comentários Finais}

Apresentamos e comparamos duas implementações de métodos de ponto interiores para redes, lineares e lineares por partes, respectivamente. Comparamos então duas estratégias para a resolução de problemas de fluxo lineares por partes:

(1) Converter o problema linear por partes em outro linear equivalente e aplicar o algoritmo para problemas lineares.

(2) Resolver o problema por um algoritmo especializado, capaz de atacar o problema linear por partes diretamente.

A investigação do método mais adequado de conversão a ser utilizado em (1) foi objeto de estudo anterior pelos autores [10]. No presente trabalho foi utilizado o $\delta$-Método, que consiste em substituir cada variável cujo custo é linear por partes por uma soma de variáveis, uma para cada intervalo em que o custo é linear. Esta transformação apresenta, entre outras, a vantagem de preservar a estrutura de rede do problema, a matriz de coeficientes do problema linear equivalente continua sendo uma matriz de incidência de uma rede.

Elaboramos uma versão do método de pontos interiores para redes lineares adaptada para a resolução de redes lineares por partes. As implementações dos algoritmos foram testadas em 114 problemas gerados aleatoriamente. Ambos os algoritmos utilizam as mesmas regras de parada, ou seja, o controle de qualidade da solução que será aceita como ótima tem o mesmo rigor em ambos os casos. Os testes comprovam a superioridade da estratégia (2), sendo os tempos de CPU aproximadamente $50 \%$ maiores na estratégia (1).

Os autores investigam atualmente a adaptação do programa $\mathrm{PCx}$, baseado em métodos de pontos interiores, para a resolução de problemas lineares para verificar se a superioridade da estratégia (2) permanece válida para problemas lineares gerais.

\section{Referências}

[1] C. Cavichia, Resolução de Problemas de Programação Linear por Partes via Algoritmos de Pontos Interiores, Tese de Doutorado, Faculdade de Engenharia Elétrica e de Computação, Unicamp, 1997.

[2] J. Czyzyk, S. Mehrotra, M. Wagner, S.J. Wright, PCx user guide, Technical Report OTC 96/01, Optimization Technology Center, 1997.

[3] K. Darby-Dowman, F.A.S. Marins, E. Senne, C. Perin, A. Machado, Algorithms for network piecewise-linear programs: a comparative study, European Journal of Operational Research 97 (1997), 183-199.

[4] J.K. Ho, Relationships among linear formulations of separable convex piecewise linear programs, Mathematical Programming Study 24 (1985), 126-140.

[5] F.A.S. Marins, C. Perin, Programação linear por partes, Produção 6 (1996), 146-163. 
[6] F.A.S. Marins, C. Perin, Algoritmos para programas em redes lineares por partes, Pesquisa Operacional 15 (1995) 67-90.

[7] F.A.S. Marins, C. Perin, An interior point approach to piecewise-linear programs, in "Anais de Resumos do Euro XV/Informs XXXIV Joint International Conference", Barcelona, Espanha, 1997.

[8] F.A.S. Marins, C. Perin, A primal-dual interior point method for network piecewise-linear programs, in "Anais de Resumos do 16th International Symposium on Mathematical Programming", Lausanne, Suíça, 1997.

[9] S. Mehotra, J. Wang, Conjugate gradient based implementation of an interior point method for network flow problems, Technical Report 95-70, Northwestern University, Evanston, IL, USA, 1995.

[10] C. Perin, F.A.S. Marins, M.P. Mello, Solução de programas lineares por partes em redes via pontos interiores, in "Anais de Resumos do XXI CNMAC", Caxambu, MG, 1998.

[11] M. Resende, G. Veiga, An efficient implementation of a network interior point method, Technical Report, AT\&T Bell Laboratories, Murray Hill, NJ, USA, 1992.

[12] Y. Zhang, Solving large-scale linear programs by interior point methods under the MATLAB environment, Technical Report, Department of Compputational and Applied Mathematics, Rice University, Houston, TX, USA, 1997. 